\title{
Prognostic value of broad-spectrum keratin clones AE1/AE3 and CAM5.2 in small cell lung cancer patients undergoing pulmonary resection
}

\author{
Andrzej Badzio1 ${ }^{\square}$, Piotr Czapiewski2,4, Adam Gorczyński², Kamila Szczepańska-Michalska3, \\ Johannes Haybaeck ${ }^{4,5,6}$, Wojciech Biernat ${ }^{2}$ and Jacek Jassem ${ }^{7}$ \\ 1Radiotherapy Center NU-Med, Elblag, Poland; ${ }^{2}$ Department of Pathology, Medical University of Gdansk, Poland; ${ }^{3}$ Copernicus Hospital, Gdansk, \\ Poland; ${ }^{4}$ Department of Pathology, Medical Faculty, Otto-von-Guericke University Magdeburg, Germany; 5 Institute of Pathology, Medical \\ University of Graz, Austria; ${ }^{6}$ Department of Pathology, Neuropathology and Molecular Pathology, Medical University Innsbruck, Austria; \\ 7Department of Oncology and Radiotherapy, Medical University of Gdansk, Poland
}

Introduction: Small cell lung carcinoma (SCLC) is an aggressive pulmonary neoplasm of neuroendocrine origin. Keratins form a large group of intermediate filaments, which are major structural proteins in epithelial cells and carcinomas. SCLC shows a wide spectrum of keratin expression, from very strong to completely negative. A prognostic role of keratin expression in SCLC is unknown. Material and Methods: Tumor tissue microarray samples from a unique series of 82 SCLC patients who underwent pulmonary resection were stained with keratin specific antibodies AE1/AE3 and CAM5.2. The percentage o1f positively stained cells and their staining pattern (diffusely membranous, partially membranous and dot-like) were evaluated. The median expression value was used for the distinction between keratin-negative and -positive patients. Overall survival in respective groups was compared using the logrank test. Multivariate Cox proportional hazards regression analysis was performed adjusting for age, gender, tumor site, tumor stage, and tumor histology. Results: edian expression of AE1/AE3 and CAM5.2 was $80 \%$ and $90 \%$, respectively. Five cases were completely negative for AE1/AE3 and three for Cam5.2. Median overall survival for patients with stronger and weaker AE1/AE3 staining was 24.7 and 13.8 months, respectively $(p=0.019)$. There was no difference in survival in relation to the CAM5.2 expression $(p=0.44)$. In multivariate analysis adjusted for CAM5.2, $\mathrm{T}$ and $\mathrm{N}$ stage, gender and age at diagnosis, stronger AE1/AE3 expression was an independent predictor of increased survival (HR $0.50 ; 95 \% \mathrm{Cl}, 0.27-$ $0.94 ; p=0.031)$. Conclusion: High expression of AE1/AE3 is a favorable prognostic factor in surgically treated SCLC. The applicability of this finding to a typical patient population treated with non-surgical methods warrants further studies.

Key words: SCLC, CKAE1/AE3, CAM5,2, prognosis

Received: 30 January, 2019; revised: 17 February, 2019; accepted: 17 February, 2019; available on-line: 22 February, 2019

曰e-mail: abadzio@gumed.edu.pl

Abbreviations: SCLC, small cell lung cancer; NSCLC, non-small cell lung cancer; $\mathrm{PCl}$, prophylactic cranial irradiation; $\mathrm{PS}$, performance status; TMA, Tissue microarray

\section{INTRODUCTION}

Small cell lung cancer (SCLC) represents around 15\% of all bronchogenic neoplasms, and is characterized by early dissemination and rapid tumor growth. Surgery used to be the standard management of patients with early disease until the late 1960 s, with very poor outcomes due to early dissemination (Kirklin et al.,, 1955). Surgery for SCLC was generally abandoned after a study of the British Medical Council had shown that fewer than $2 \%$ of patients survived for more than two years after resection (Fox \& Scadding, 1973). Subsequently, after cyclophosphamide and a number of other cytotoxic drugs had been found to improve survival in SCLC, chemotherapy became the mainstay of SCLC treatment (Green et al.,, 1969; Morstyn et al., 1984). However, surgery has remained an option for highly selected low stage patients, with relatively good outcome (Hamilton et al., 2016; Yang et al., 2017). Despite the high response rates to chemotherapy, prognosis for SCLC patients has remained poor, with a median survival of only 12-14 and 9 months, for limited and extensive disease, respectively(Waqar \& Morgensztern, 2017). Further studies on the biology of this disease are warranted to develop new therapeutic strategies. Numerous studies suggest that SCLC is not a uniform disease (Grand et al., 2013; Sabari et al., 2017), including subsets of patients with different characteristics (Yashiro \& Matsuoka, 2015).

Keratins are a large family of proteins expressed in the epithelial and epidermal cells where they form a cytoskeletal structure. Keratins form intermediate filaments which constitute a characteristic feature of epithelial cells. The keratin family includes more than 80 types of heterodimer-forming proteins, including one molecule of type I and another of type II keratin (Karantza, 2011). Normal epithelial cells usually show a specific keratin profile typical for various histological types of epithelia. Carcinomas usually retain the keratin expression pattern of the original cell, and in many of them the aberrant expression of some keratins is associated with poor prognosis (Czapiewski et al., 2016; Fillies et al., 2006; Govaere et al., 2014; Makino et al., 2009; Oue et al., 2012).

SCLCs express numerous types of keratins and show substantial variability in the intensity of staining. Despite their epithelial origin, some SCLCs are keratin-negative (Righi et al., 2017), which may sometimes cause clinically relevant diagnostic errors. The expression of keratins in SCLC is significantly lower than in the large cell neuroendocrine carcinoma(Nagashio et al., 2010) and nonsmall cell carcinoma (NSCLC) (Nobuhiro Kanaji et al., 2011), and the prognostic role of this feature in SCLC has not been extensively studied. 
AE1/AE3 is the most widely used antibody cocktail to detect cytokeratin expression. AE1 detects the high molecular weight keratins 10,14, 15 and 16, and the low molecular weight cytokeratin 19. Clone AE3 detects type II high molecular weight keratins 1, 2, 3, 4, 5 and 6, and the low molecular weight keratins 7 and 8 (Ordóñez, 2013). CAM5.2, another antibody used in this setting, reacts only with Ks 7 and 8 (Hsu et al., 2010).

Our patient population, one of the largest series of this type described in the literature, was unique as it included SCLC patients who underwent pulmonary surgical resection.

Our study focused on the occurrence and prognostic role of the broad-spectrum keratin expression in SCLC.

Patient population. This study used archival formalin-fixed, paraffin-embedded tumor samples from 97 limited disease SCLC patients who had undergone lung tumor resection over a period of 20 years, between 1982 and 2002. In this unique series, surgery was the first treatment option due to difficulties in preoperatively obtaining a reliable biopsy material for histopathological examination, and SCLC was only diagnosed at the time of resection. All patients underwent tumor resection and mediastinal lymphadenectomy and were administered one of the standard chemotherapy schemes. Only 4 patients received prophylactic cranial irradiation (PCI) and 2 patients received thoracic irradiation after surgery. Patient Performance Status (PS) was assessed in 35 patients, patient $\mathrm{T}$ and $\mathrm{N}$ status was assessed based on surgical pathology report.

Tissue microarray preparation. On each formalin-fixed, paraffin-embedded block, a morphologically representative SCLC area was microscopically identified by an experienced pathologist. To this end, a hematoxylin and eosin-stained section on a glass slide served as a guide. Tissue microarray (TMA) was constructed using manual TMA I Beecher Instrument. For each patient, from two up to four $1.5 \mathrm{~mm}$ cores were taken from various tumor areas.

Immunohistochemistry. Immunohistochemistry was performed using ready to use primary monoclonal antibody against cytokeratins (clone AE1/AE3, DAKO,



Figure 1. Overall survival for high and low AE1/AE3 expression cases.

Glostrup, Denmark; clone CAM5.2, Ventana, Tuscon, Arizona, USA) on automatic detection platforms (Autostainer Link 48, (Dako) and Benchmark GX (Cell Signaling Technology, Danvers, MA, USA, Ventana). Pretreatment with heat-induced epitope retrieval was performed using Dako PT Link (Code PT100/PT101) with EnVisionTM FLEX Target Retrieval Solution, High $\mathrm{pH}(\mathrm{pH}$. 9.0, Code K8004).

Positively stained cancer cells were quantified, and the results were provided as percentages. In addition, the prevalent staining pattern was also evaluated: dotlike for cytoplasmic staining in the peri-Golgi apparatus (type 1), partially membranous for membranous staining of incomplete membrane circumference (type 2), and completely membranous circumferential for diffuse staining of the whole cell membrane circumference (type 3). Representative cases of the above-mentioned staining patterns are shown in Fig. 1. All cases were evaluated independently for each staining by two experienced pathologists (P.C. and A.G.).

Statistical analysis. Stata 8 was used for all statistical analyses. Group comparisons were conducted using two-sided Pearson's chi-square tests for categorical data

Table 1. Clinicopathological characteristics of patients, according to the staining intensity

\begin{tabular}{|c|c|c|c|c|c|c|}
\hline Variable & $\begin{array}{l}\text { CKAE1/AE3 } \\
\text { (positive) }\end{array}$ & $\begin{array}{c}\text { CKAE1/AE3 } \\
\text { (negative) }\end{array}$ & $p$ value & $\begin{array}{l}\text { CAM5.2 } \\
\text { (positive) }\end{array}$ & $\begin{array}{l}\text { CAM5.2 } \\
\text { (negative) }\end{array}$ & $p$ value \\
\hline Age (Average) & 56.6 & 59.6 & 0.11 & 57.7 & 573 & 0.85 \\
\hline \multicolumn{7}{|l|}{ Sex (\%) } \\
\hline Men & $29(75)$ & $28(74)$ & & $23(70)$ & $33(75)$ & \\
\hline Women & $10(25)$ & $10(26)$ & 0.95 & $10(30)$ & $11(25)$ & 0.26 \\
\hline Tumor size (mean) & $4.3 \mathrm{~cm}$ & $4.9 \mathrm{~cm}$ & 0.29 & $4.5 \mathrm{~cm}$ & $4.6 \mathrm{~cm}$ & 0.84 \\
\hline \multicolumn{7}{|l|}{ pT stage (\%) } \\
\hline & $6(15)$ & $3(8)$ & & $5(15)$ & $4(9)$ & \\
\hline & $20(51)$ & $22(58)$ & & $27(49)$ & $27(61)$ & \\
\hline T3 & $8(21)$ & $7(18)$ & & $9(15)$ & $9(21)$ & \\
\hline T4 & $5(13)$ & $6(16)$ & 0.74 & $7(21)$ & $4(9)$ & 0.33 \\
\hline \multicolumn{7}{|l|}{ pN stage (\%) } \\
\hline No & $15(38)$ & $20(52)$ & & $15(45)$ & $19(44)$ & \\
\hline N1 & $7(18)$ & $6(16)$ & & $4(12)$ & $9(21)$ & \\
\hline N2 & $17(44)$ & $11(29)$ & 0.38 & $14(43)$ & $15(34)$ & 0.59 \\
\hline \multicolumn{7}{|l|}{ Karnofsky Status (\%) } \\
\hline 100 & $2(12)$ & $4(24)$ & & $3(16)$ & $3(19)$ & \\
\hline 90 & $7(44)$ & $9(53)$ & & $11(58)$ & $7(44)$ & \\
\hline 80 & 7 (44) & $4(23)$ & 0.42 & $5(26)$ & $6(37)$ & 0.695 \\
\hline
\end{tabular}




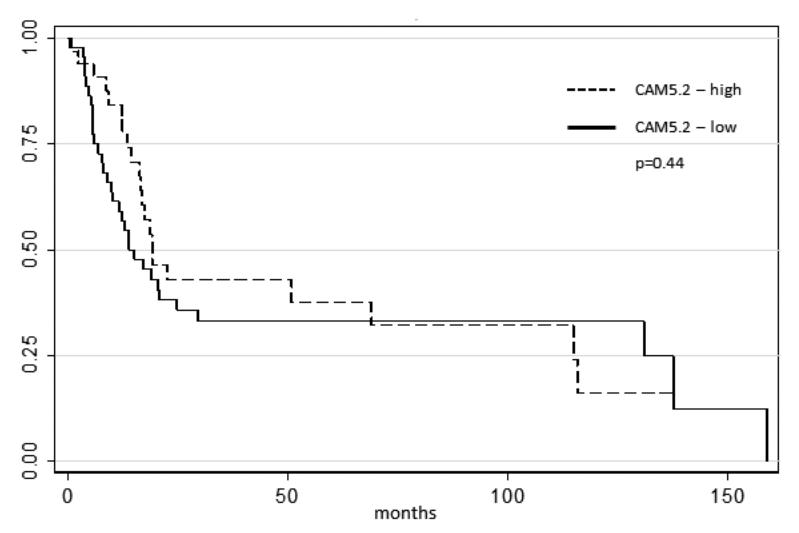

Figure 2. Overall survival for high and low CAM 5.2 expression cases

and two-sided Student's $t$-tests for continuous data. Associations between continuous measures were compared using Pearson's product-moment correlation coefficient. Overall survival was calculated as time from the surgery to the date of last follow-up visit or death, and plotted with 95\% confidence intervals using the Kaplan-Meier method. Outcomes in particular groups were compared using the log-rank test. Multivariate Cox proportional hazards regression analysis was performed adjusting for age, gender, tumor site, tumor stage, and tumor histology. All tests were considered statistically significant at $p<0.05$.

\section{RESULTS}

\section{Frequency of AE1/AE3 and CAM5.2 expression}

AE1/AE3 and CAM5.2 expression was evaluated in all 82 patients. The average and median expression of AE1/AE3 was $66 \%$ and $80 \%$, respectively. A threshold of $80 \%$ was used for the distinction between negative and positive cases, resulting in $40(49 \%)$ and $42(51 \%)$ of low and high expression cases, respectively. The average expression of CAM5.2 was $75 \%$, and the median was $90 \%$. With a threshold of $90 \%, 45(55 \%)$ and 37 $(45 \%)$ patients were assigned to low and high expression groups, respectively. AE1/AE3 and CAM5.2 expression was not correlated with age, sex, tumor size, $\mathrm{T}$ stage, $\mathrm{N}$ stage and PS (Table 1).

\section{Keratin staining patterns}

Five cases were completely negative for AE1/AE3. Among the positive cases, seven $(9 \%)$ showed predominantly type 1 staining, $29(38 \%)$ predominantly type 2 , and $41(53 \%)$ predominantly type 3 staining. For CAM 5.2, three patients $(3.5 \%)$ were completely negative; among positive cases, eight $(10 \%)$ showed predominantly type 1 staining, $41(52 \%)$ type 2 , and $30(38 \%)$ type 3 .

\section{Keratin expression and prognosis}

Median overall survival time for patients with stronger and weaker AE1/AE3 was 24.7 and 13.8 months, respectively ( $p=0.019$; Fig. 1$)$. There was no difference in survival in relation to the CAM5.2 expression $(p=0.44$, Fig. 2). Staining patterns of both AE1/AE3 and CAM5.2 were not predictive for survival $(p=0.38$ and 0.74 , respectively, Figs. 3 and 4). In multivariate analysis adjusted for CAM5.2, the $\mathrm{T}$ and $\mathrm{N}$ stage, gender and age



Figure 3. Overall survival according to the staining pattern for AE1/AE3

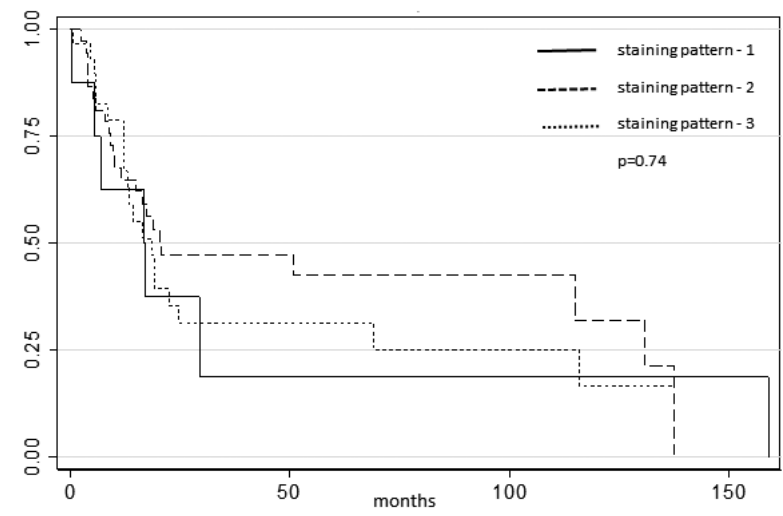

Figure 4. Overall survival according to the staining pattern for CAM5.2

at diagnosis, stronger AE1/AE3 expression was the only independent predictor of increased survival (HR 0.50; 95\% CI, 0.27-0.94; $p=0.031$ ).

\section{DISCUSSION}

To the best of our knowledge, we are the first to show that high expression of broad-spectrum keratin, corresponding to the high content of the keratinic intermediate filament, is a favorable prognostic factor in SCLC. Keratin expression in SCLC is weaker than in other lung cancer types, and the lack of its expression does not exclude the diagnosis of SCLC. The pathological data are corroborated by in vitro studies, which had shown that the levels of keratins expressed in SCLC cell lines were significantly lower than in the NSCLC lines (Kanaji et al., 2011). Among seven SCLC cell lines tested, only three (H69, H209 and Lu134) displayed K8 expression, and H209 were additionally characterized by K18 and K19 expression. Other cell lines (Lu135, Lu165, TO1019 and MN1112) had barely detectable keratin levels of these proteins. In another study, the expression levels of K7, K8, K18 and K19 were4.6, 27, 17 and 3.3 times higher in the large cell neuroendocrine carcinoma cell line LCN1 than in the SCLC cell line N231, respectively (Nagashio et al., 2010). In the same study, an immunohistochemical analysis of human tissue specimens revealed a significant difference in the frequency of K7 reaction $(63 \%$ vs. $90 \%)$, whereas the reaction frequency of the remaining keratins was similar. Of note, for all 
keratins, the staining score was much lower for SCLC (Nagashio et al., 2010). This observation may explain the difference in the prognostic role between AE1/AE3 and CAM5.2 in our study. Both keratin clones are widely used in surgical pathology departments. CAM5.2 contains $\mathrm{K} 8$ and, to a lesser extent, $\mathrm{K} 7$, whereas AE1/AE3 is composed of antibodies against Ks 1-8, 10, 14-16 and 19 (Ordóñez, 2013). Importantly, our population of patients is unique as it included patients who underwent pulmonary resection. In clinical practice, the vast majority of SCLC patients is treated with chemotherapy with or without radiotherapy, and it is unknown whether the favorable prognostic role of AE1/AE3 is equally favorable for non-operated SCLC patients. Data on the prognostic impact of keratin expression in SCLC is scarce. One study reported shorter progression-free survival and overall survival in SCLC patients with positive mRNA for K19 in the peripheral blood (Shi et al., 2013), and another study showed an adverse prognostic impact of Cyfra21-1 (a fragment of K19) in the blood samples (Ando et al., 2004). Although the prognostic impact of the keratins' level was confirmed by multivariate analysis in both studies, they might have been still more related to the tumor burden and not to the keratin level in the tumor tissue. In contrast to the above-mentioned studies, our results suggest better prognosis for patients with positive AE1/AE3 staining, composed of antibodies against various keratins. Notably, however, our study included a highly selected subset of SCLC patients, and the question of generality of those results warrants further studies. As we did not analyze particular types of keratins, we were also unable to evaluate their potentially different prognostic role. For example, in squamous cell carcinoma of the esophagus, the negative prognostic role of $\mathrm{K} 18$ was much stronger than that of $\mathrm{K} 8$, despite the $82.4 \%$ concordance rate between the staining intensities of both. Further analyses of particular types of keratins, for example K7, K8, K18 or K19 in SCLC, may answer these questions. We are aware of the limitations of this study, in particular of its retrospective character and a long period of material collection. Within this time period, staging procedures and types of chemotherapy were subjected to substantial changes, which might have impacted patient survival. Finally, as all patients in this study were treated with surgery, the clinical relevance of our findings in SCLC patients treated with standard chemotherapy and radiation may be limited.

In conclusion, this study has shown that low AE1/ AE3 expression is an adverse prognostic factor in SCLC patients undergoing pulmonary resection.

\section{REFERENCES}

Ando S, Suzuki M, Yamamoto N, Iida T, Kimura H (2004) The prognostic value of both neuron-specific enolase (NSE) and Cyfra21-1 in small cell lung cancer. Anticancer Res 24: 1941-1946. PMID: 15274381

Czapiewski P, Bobowicz M, Pęksa R, Skrzypski M, Gorczyński A, Szczepańska-Michalska K, Korwat A, Jankowski M, Zegarski W, Szulgo-Paczkowska A, Polec T, Piatek M, Skokowski J, Haybaeck J, Żaczek A, Biernat W (2016) Keratin 7 expression in lymph node metastases but not in the primary tumour correlates with distant metastases and poor prognosis in colon carcinoma. Polish J Pathol 67: 228-234. https://doi.org/10.5114/pip.2016.63774

Fillies T, Werkmeister R, Packeisen J, Brandt B, Morin P, Weingart D, Joos U, Buerger H (2006) Cytokeratin 8/18 expression indicates a poor prognosis in squamous cell carcinomas of the oral cavity. BMC Cancer 6:10. https://doi.org/10.1186/1471-2407-6-10
Fox W, Scadding JG (1973) Medical research council comparative trial of surgery and radiotherapy for primary treatment of small-celled or oat-celled carcinoma of bronchus. Ten-Year Follow-Up. The Lancet 302: 63-65. https://doi.org/10.1016/S0140-6736(73)93260-1

Govaere O, Komuta M, Berkers J, Spee B, Janssen C, de Luca F, Katoonizadeh A, Wouters J, van Kempen LC, Durnez A, Verslype C, De Kock J, Rogiers V, van Grunsven LA, Topal B, Pirenne J, Vankelecom H, Nevens F, van den Oord J, Pinzani M, Roskams T (2014) Keratin 19: A key role player in the invasion of human hepatocellular carcinomas." Gut 63: 674-685. https://doi.org/10.1136/ gutjnl-2012-304351

Grand B, Cazes A, Mordant P, Foucault C, Dujon A, Guillevin EF, Barthes Fle P, Riquet M (2013) High grade neuroendocrine lung tumors: pathological characteristics, surgical management and prognostic implications. Lung Cancer (Amsterdam, Netherlands) 81: 404-409. https://doi.org/10.1016/j.lungcan.2013.05.008

Green RA, Humphrey E, Close H, Patno ME (1969) Alkylating agents in bronchogenic carcinoma. Am J Med 46: 516-525.

Hamilton G, Rath B, Ulsperger E (2016) A review of the role of surgery for small cell lung cancer and the potential prognostic value of enumeration of circulating tumor cells. Eur JSurg Oncol 42: 12961302. https://doi.org/10.1016/j.ejso.2016.04.063

Hsu JD, Yao CC, Han LW, Han CP (2010) CAM5.2 Is not identical to cytokeratins 8 and 18. Am J Clin Pathol 133: 514. https://doi. org/10.1309/AJCPAKB6JEBVMX5U

Kanaji N, Bandoh S, Ishii T, Fujita J, Ishida T, Matsunaga T, Kubo A. (2011) Cytokeratins negatively regulate the invasive potential of lung cancer cell lines. Oncol Rep 26: 763-768. https://doi.org/10.3892/ or. 2011.1357

Karantza V (2011) Keratins in health and cancer: more than mere epithelial cell markers. Oncogene 30: 127-138. https://doi.org/10.1038/ onc. 2010.456

Kirklin JW, McDonald JR, Clagett OT, Moersch HJ, Gage RP (1955) Bronchogenic carcinoma: cell type and other factors relating to prognosis. Surg Gynecol Obstet 100: 429-438. PMID: 14373336

Makino T, Yamasaki M, Takeno A, Shirakawa M, Miyata H, Takiguchi S, Nakajima K, Fujiwara Y, Nishida T, Matsuura N, Mori M, Doki Y (2009) Cytokeratins 18 and 8 are poor prognostic markers in patients with squamous cell carcinoma of the oesophagus. Brit J Cancer 101: 1298-1306. https://doi.org/10.1038/sj.bjc.6605313

Morstyn G, Ihde DC, Lichter AS, Bunn PA, Carney DN, Glatstein E, Minna JD (1984) Small cell lung cancer 1973-1983: early progress and recent obstacles. Int J Rad Oncol Biol Phys 10: 515-539. https:// doi.org/10.1016/0360-3016(84)90032-4

Nagashio R, Sato Y, Matsumoto T, Kageyama T, Satoh Y, Ryuge S, Masuda N, Jiang SX, Okayasu I (2010) Significant high expression of cytokeratins $7,8,18,19$ in pulmonary large cell neuroendocrine carcinomas, compared to small cell lung carcinomas. Pathol Int 60: 71-77. https://doi.org/10.1111/j.1440-1827.2009.02487.x

Ordóñez NG (2013) Broad-spectrum immunohistochemical epithelial markers: a review. Hum Pathol 44: 1195-1215. https://doi. org/10.1016/j.humpath.2012.11.016

Oue N, Noguchi T, Anami K, Kitano S, Sakamoto N, Sentani K, Uraoka N, Aoyagi K, Yoshida T, Sasaki H, Yasui W (2012) Cytokeratin 7 is a predictive marker for survival in patients with esophageal squamous cell carcinoma. Ann Surg Oncol 19: 1902-1910. https:// doi.org/10.1245/s10434-011-2175-4

Righi L, Gatti G, Volante M, Papotti M (2017) Lung neuroendocrine tumors: pathological characteristics. J Thorac Dis 9: S1442-S1447. https://doi.org/10.21037/jtd.2017.01.59

Sabari JK, Lok BH, Laird JH, Poirier JT, Rudin CM (2017) Unravelling the Biology of SCLC: Implications for Therapy. Nat Rev Clin Oncol 14: 549-561. https://doi.org/10.1038/nrclinonc.2017.71

Shi WL, Li J, Du YJ, Zhu WF, Wu Y, Hu YM, Chen YC (2013) CK-19 mRNA-positive cells in peripheral blood predict treatment efficacy and survival in small-cell lung cancer patients. Med Oncol (Northwood, London, England) 30: 755. https://doi.org/10.1007/ s12032-013-0755-9

Waqar SN, Morgensztern D (2017) Treatment advances in small cell lung cancer (SCLC). Pharmacol Therapeutics 180: 16-23. https://doi. org/10.1016/j.pharmthera.2017.06.002

Yang CJ, Chan DY, Shah SA, Yerokun BA, Wang XF, D'Amico TA, Berry MF, Harpole DH Jr (2018) Long-term survival after surgery compared with concurrent chemoradiation for node-negative small cell lung cancer. Ann Surg 268: 1105-1112 https://doi.org/10.1097/ SLA.0000000000002287

Yashiro M, Matsuoka T (2015) Sentinel node navigation surgery for gastric cancer: overview and perspective. World J Gastrointest Surg 7: 1-9. https://doi.org/10.4240/wjgs.v7.i1.1 